\title{
Warm Molecular Sphere around Red Supergiant Stars- A Missing Link between the Photosphere and Masering Water Clouds in the Circumstellar Envelope
}

\author{
Takashi Tsuji \\ Institute of Astronomy, School of Science, The University of Tokyo \\ 2-21-1, Osawa, Mitaka, Tokyo, 181-0015 Japan
}

Interferometry of red supergiants before 2000: The M2 supergiant Betelgeuse ( $\alpha$ Ori) is the first star whose angular diameter was measured by the Michelson stellar interferometer (Michelson \& Pease 1921). Since then, however, we had to wait half a century before we witnessed the renaissance of interferometric observations in optical astronomy with novel methods such as speckle interferometry (Laberie 1970). Michelson's classical method was also extended to the infrared regime (e.g. McCarthy et al. 1977). The initial results served not only to compare stellar temperature scale with the measured angular diameters but also to probe scattering and thermal emission in the dust envelope around red supergiant stars (e.g. Tsuji 1978,1979). Interferometry has been more active in the radio domain: In connection with our subject, VLBI observations revealed many masering water clouds around red supergiants such as VY CMa (Imai et al. 1997) and S Per (Richards et al. 1999), but their origin in unknown. Also, radio observation of Betelgeuse with the VLA (Lim et al. 1998) revealed the presence of a new component of modest temperatures $\left(T_{\text {ex }} \lesssim 3500 \mathrm{~K}\right)$ over the same height range as the classical chromosphere $\left(T_{\mathrm{ex}} \gtrsim 5000 \mathrm{~K}\right)$, but again the nature of the new component remains obscure.

Recent space infrared spectroscopy: Stellar spectroscopy has a longer history than interferometry, but we are still far from understanding stellar spectra in full detail. For example, water was discovered in the early M supergiants $\alpha$ Ori and $\mu$ Cep more than 35 years ago by the pioneering space infrared spectroscopy with the balloon-borne Stratoscope II (Woolf et al.1964; Danielson et al.1965). However, this important discovery was so unexpected at that time (and even today) that it was misinterpreted and overlooked for a long time. Recently, we showed that the Stratoscope observers in fact discovered water in these supergiants not necessarily very cool but that the water detected by them may not originate in the photosphere (Tsuji 2000a). Also, water absorption bands were detected in the ISO spectra of the early M supergiants of the $h+\chi$ Persei clusters (Tsuji et al.1997/8) as well as in the mid infrared spectra of Betelgeuse and Antares (Jennings \& Sada 1998). Thus, the presence of water in the early $M$ supergiants is well confirmed, against the general belief that water exists only in the very cool stars such as Mira variables. Finally, we detected water in emission at 6 and $40 \mu \mathrm{m}$ in the ISO spectrum of $\mu \mathrm{Cep}$, and we confirmed that the water originates in the outer atmosphere rather than in the starspots or in the temperature inhomogeneities due to the large convective 
cells. We interpret the results, together with the Stratoscope data, as due to a rather warm $\left(T_{\mathrm{ex}} \approx 1500 \mathrm{~K}\right)$ and optically thick $\left(\tau \approx 10^{3}\right.$ in $\mathrm{H}_{2} \mathrm{O}$ lines or $N_{\text {col }}\left(\mathrm{H}_{2} \mathrm{O}\right) \approx 3 \times 10^{20} / \mathrm{cm}^{2}$ ) water sphere with inner radius of about $2 R_{*}$ (Tsuji $2000 \mathrm{~b}$ ). We refer to the warm water sphere as MOLsphere for simplicity. Thus, the outer atmosphere of red supergiant stars should have a complicated hybrid and/or hierarchical structure consisting of the new MOLsphere $\left(T_{\mathrm{ex}} \approx 10^{3} \mathrm{~K}\right)$ together with the rather hot chromosphere $\left(T_{\mathrm{ex}} \approx 10^{4} \mathrm{~K}\right)$ and the cold expanding gas-dust shells $\left(T_{\mathrm{ex}} \approx 10^{2} \mathrm{~K}\right)$ known so far.

Future (space infrared) interferometry: The MOLsphere is not yet observed with the interferometry, despite its marvelous success in the whole spectral region. This is because the infrared region, which best provides the possibility to detect MOLsphere, was mostly unaccessible from the ground. With the high resolution space interferometer, the MOLsphere will easily be "seen" by molecular lines, especially by the optically thick $\mathrm{H}_{2} \mathrm{O}$ lines. The MOLsphere may probably be the birth place of the masering water clouds, and also be closely related to the new component seen by the VLA around Betelgeuse. All these problems can be clarified once the detailed structure of the MOLsphere is observed with the high spatial resolution. For this purpose, $\mu$ Cep may be a possible candidate, since it is also a $\mathrm{H}_{2} \mathrm{O}$ maser source (González-Alfonso et al. 1998). The more difficult problem is the origin of the MOLsphere itself. In fact, no mechanism is known to transport and to sustain such a large amount of mass (total mass of the MOLsphere is about $10^{-4} M_{\odot}$ ) above the photosphere in the outer atmosphere. To understand the exact nature of the MOLsphere and further to develop a unified model of the outer atmosphere of red supergiant stars, high resolution spatial interferometry by spectral lines or spatially resolved high resolution spectroscopy, preferably from space, will be essential.

\section{References}

Danielson, R. E., Woolf, N. J., \& Gaustad, J. E. 1965, ApJ, 141, 116

González-Alfonso, E., Cernicharo, J., Alcolea, J., \& Orlandi, M. A. 1998, A\&A, 334, 1016

Imai, H., Sasao, T., Kameya, O., Miyoshi, M. et al. 1997, A\&A, 317, L67

Lim, J., Carilli, C. L., White, S. M., Beasley, A. J. et al. 1998, Nature, 392, 575

Jennings, D. E., \& Sada, P. V. 1998, Science, 279, 844

Laberie, A. 1970, A\&A, 6, 85

McCarthy, D. W., Low, F. J., \& Howell, R. 1977, ApJ, 214, L85

Michelson, A. A., \& Pease, F. G. 1921, ApJ, 53, 249

Richards, A. M. S., Yates, J. A., \& Cohen, R. J. 1999, MNRAS, 306, 954

Tsuji, T. 1978, PASJ, 30, 435

Tsuji, T. 1979, PASJ, 31, 43

Tsuji, T. 2000a, ApJ, 538, 801

Tsuji, T. 2000b, ApJ, 540, L99

Tsuji, T., Ohnaka, K., Aoki, W., \& Yamamura, I. 1997/8, Ap\&SS, 255, 293

Woolf, N. J., Schwarzschild, M., \& Rose, W. K. 1964, ApJ, 140, 833 